\title{
EXTREMAL PROPERTIES OF REAL AXIALLY SYMMETRIC HARMONIC FUNCTIONS IN $E^{3}$
}

\author{
PETER A. MCCOY
}

This paper is dedicated to the memory of Professor Stefan Bergman

from whose work we have learned so much

\begin{abstract}
The set $\mathcal{H}$ consists of all real harmonic functions defined by $U(r, \theta)=\sum_{k-0}^{\infty} a_{k} r^{k} P_{k}(\cos \theta)$ which are regular in $\Sigma$, the open unit sphere about the origin $E^{3}$. Two problems arise concerning $\mathcal{X}$ and a subset $\mathcal{X}$. whose members $U$ have the first $n+1$ coefficients $a_{0}, \ldots, a_{n}$ specified. (1) For $U \in \mathcal{X}$, determine $I(U)=\inf \{U(r, \theta) \mid(r, \theta) \in \Sigma\}$ as the limit of a monotone sequence of constants $\left\{\lambda_{k}\left(a_{0}, \ldots, a_{k}\right)\right\}_{k=0}^{\infty}$ which can be computed algebraically. (2) Find $U_{0} \in \mathcal{K}$ and the constant

$$
\lambda_{n}\left(a_{0}, \ldots, a_{n}\right)=\operatorname{Sup}\left\{I(U) \mid U \in \mathcal{K}_{*}\right\}=I\left(U_{0}\right) .
$$

These are answered by means of the Bergman Integral Operator Method and applications of the Methods of Ascent and Descent to the classical Carathéodory-Fejér problem regarding extremal properties of harmonic functions in $E^{2}$.
\end{abstract}

1. Introduction. In the theory of analytic functions

$$
f(z)=a_{0}+2 \sum_{k=1}^{\infty} a_{k} z^{k}
$$

of one complex variable, the unique relation between $f$ and its Taylor's coefficients has been exploited to provide definitive characterizations of three fundamental properties of $f$;

(i) the location and nature of the singularities [Hadamard, Mandelbrojt (see [3])]

(ii) the distribution of values [Cauchy, Carathéodory-Toeplitz and Schur, (see [7], [11])]

(iii) and extremal properties of certain functionals defined for $f$ analytic in the open unit disk $D$ about the origin [Carathéodory-Fejér, (see [6])]. These are translated to statements on real harmonic functions (HF)

$$
u(x, \rho)=a_{0}+2 \sum_{k=1}^{\infty} a_{k} u_{k}(x, \rho),
$$

Presented to the Society, November 19, 1976; received by the editors November 12, 1976 and, in revised form, March 10, 1977.

AMS (MOS) subject classifications (1970). Primary 35B05, 35C10; Secondary 30A08.

Key words and phrases. Bergman and Gilbert's integral operators, extremal properties of harmonic functions, Carathéodory-Fejér theorems.

(1) American Mathematical Society 1978 


$$
(x+i \rho)^{k}=u_{k}(x, \rho)+i v_{k}(x, \rho)
$$

in $E^{2}$ by taking the real part of the associated analytic function $f(x+i \rho)$. In $E^{3}$, one anticipates that similar characterizations of axially symmetric harmonic functions (AHF)

$$
\begin{aligned}
U(x, \rho) & =a_{0}+2 \sum_{k=1}^{\infty} a_{k} U_{k}(x, \rho), \\
U_{k}(x, \rho) & =\left(x^{2}+\rho^{2}\right)^{k / 2} P_{k}\left(x / \sqrt{x^{2}+\rho^{2}}\right),
\end{aligned}
$$

$(x, y, z) \in E^{3}, y^{2}+z^{2}=\rho^{2}, x^{2}+\rho^{2}=r^{2}, \cos \theta=x r^{-1}$ require operators whose role is analogous to that of the operator Re which represents a HF in $E^{2}$ for which a specific property is sought as the transform of an analytic function whose corresponding property is known. Application of function theoretic methods to integral representations of AHF based on the LaPlace type integral for the zonal harmonics (1.5) verified this for the first two properties.

A definitive characterization of the singularities of AHF is provided by Gilbert's theorems [4], [5] where integral operators and the "Envelope Method" extended the classical Hadamard and Mandelbrojt theorems. Moreover, an extensive literature on the singularity problem for other types of orthogonal expansions may be found in [2] and [4], [5].

The distribution of values of AHF are generalizations of the theorems in (ii). This was shown by M. Marden [8] and the author [9], [10] who applied convexity arguments from the analytic theory of polynomials of one complex variable to Bergman-Whittaker [1] and Gilbert type [4] integral representations of AHF.

This paper establishes that known properties of the functional $i(u)=$ $\lim _{r \uparrow 1} i_{r}(u)$

$$
i_{r}(u)=\inf \left\{u(x, \rho) \mid x^{2}+\rho^{2}<r^{2}\right\}
$$

defined for $u \in h$, the class of real HF in $D$, also characterize the functional $I(U)=\lim _{r \uparrow 1} I_{r}(U)$,

$$
I_{r}(U)=\inf \left\{U(x, \rho) \mid x^{2}+\rho^{2}<r^{2}\right\}
$$

defined for $U \in \mathcal{H}$, the class of real AHF in the open unit sphere about the origin in $E^{3}$. Basic to this are the integral operators which we now consider.

2. Basic formulas. The operators employed here, with the Bergman, Gilbert and Whittaker operators, are based on the LaPlace integral for the zonal harmonics which we write as

$$
U_{k}(x, \rho)=\frac{1}{2 \pi} \int_{0}^{2 \pi}\left[u_{k}(x, \rho \cos t)+i v_{k}(x, \rho \cos t)\right] d t .
$$

Visibly $v_{k}$, the conjugate harmonic function to $u_{k}$, is in the null space of the operator. This suggests the operator $U(x, \rho)=W[u(x, \rho)]$ mapping (1.2) onto (1.4) by 


$$
U(x, \rho)=\frac{1}{2 \pi} \int_{0}^{2 \pi} u(x, \rho \cos t) d t .
$$

Evidently, if $u$ is harmonic for $x^{2}+\rho^{2}<R^{2}$, the same is true of $U$.

The inverse operator uses orthogonality of the Legendre polynomials to relate the harmonic polynomials by the transform

$$
\begin{gathered}
u_{k}(x, \rho)=\int_{-1}^{1} U_{k}\left(r, r\left(1-\eta^{2}\right)^{1 / 2}\right) d \nu, \\
d \nu=K\left(x r^{-1}, \rho r^{-1}, \eta\right) d \eta
\end{gathered}
$$

whose kernel $K\left(x r^{-1}, \rho r^{-1}, \eta\right)=\operatorname{Re} K(z, \eta) / 2$ corresponds to the generating function

$$
K(z, \eta)=\sum_{k=0}^{\infty}(2 k+1) z^{k} P_{k}(\eta)=\left(1-z^{2}\right) /\left(1-2 \eta z+z^{2}\right)^{3 / 2}
$$

[1, p. 5] for the ultraspherical polynomials $C_{k}^{1 / 2}(\eta)=P_{k}(\eta)[1$, p. 24] with complex $z=(x+i \rho) r^{-1}=\operatorname{Re}[R \exp (i \phi)]$. The kernel is nonsingular if $R<$ 1. Consequently, the inversion formula $u(x, \rho)=W^{-1}[U(x, \rho)]$ associating (1.2) and (1.4) by

$$
u(x, \rho)=\int_{-1}^{1} U\left(r, r\left(1-\eta^{2}\right)^{1 / 2}\right) d \nu\left(x r^{-1}, \rho r^{-1}, \eta\right)
$$

represents a HF in $x^{2}+\rho^{2}<R^{2}$ where $U$ is harmonic in $r<R$. By construction, each real AHF is represented uniquely as the transform of a real HF and conversely. By applying definitions $[1$, p. $21 ;$ p. 7$] C_{k}^{1 / 2}(\eta)=$ $P_{k}^{(0,0)}(\eta), \quad P_{k}^{(0,0)}(1)=1$ and $P_{k}^{(-1 / 2,-1 / 2)}(\cos \theta) / P_{k}^{(-1 / 2,-1 / 2)}(1)=\cos k \theta$ to Theorem $3.3[1$, p. 25] one writes the kernel as

$$
\begin{gathered}
K(z, \eta)=\int_{-1}^{1} T\left(z, \eta^{\prime}\right) d \mu_{\eta}\left(\eta^{\prime}\right), d \mu_{\eta}\left(\eta^{\prime}\right) \geqslant 0, \\
\operatorname{Re}[T(z, \cos \theta)]=\sum_{k=0}^{\infty}(k+1 / 2) R^{k} \cos k \phi \cos k \theta .
\end{gathered}
$$

However, (2.8) being the generalized translation [1, p. 34, Theorem 4] of

$$
\operatorname{Re}[T(z, 1)]=\frac{\left(1-R^{2}\right)(1-R \cos \phi)-2 R^{2}\left(\sin ^{2} \phi\right)}{\left|1-R^{2}+2 R \cos \phi\right|^{2}},
$$

a nonnegative function for $0 \leqslant \phi \leqslant 2 \pi, 0 \leqslant R \leqslant R_{0}, R_{0}$ being the smallest positive zero of $R^{3}-3 R^{2}+1=0$, is necessarily nonnegative for $0 \leqslant \theta$, $\phi \leqslant 2 \pi, 0 \leqslant R \leqslant R_{0}$ as is the kernel by (2.7). We now turn to

3. The extremal properties. Our first result, an analogy with CarathéodoryFejér [6, pp. 147, 151], concerns the infimum of AHF in $\Sigma$.

THEOREM 1. Let $U \in \mathcal{H}$ be expanded as

$$
U(x, \rho)=a_{0}+2 \sum_{k=1}^{\infty} a_{k} U_{k}(x, \rho)
$$


and $\lambda_{k}=\lambda_{k}\left(a_{0}, \ldots, a_{k}\right)$ be the smallest eigenvalue of the Toeplitz matrix $T_{k}$

$$
T_{k}\left(a_{0}, \ldots, a_{k}\right)=\left(\begin{array}{cccc}
a_{0} & a_{1} & \ldots & a_{k} \\
a_{1} & a_{0} & \cdots & a_{k-1} \\
\vdots & & & \\
a_{k} & a_{k-1} & \cdots & a_{0}
\end{array}\right)
$$

then the sequence $\left\{\lambda_{k}\right\}_{k=0}^{\infty}$ is monotone decreasing and

$$
I(U)=\lim _{k \rightarrow \infty} \lambda_{k} .
$$

Proof. Let $U \in \mathcal{H}$ and $u \in h$ such that $U(x, \rho)=W[u(x, \rho)]$. Then since $W[1]=1, U(x, \rho) \geqslant i(u), x^{2}+\rho^{2}<1$ and

$$
I(U) \geqslant i(u) \text {. }
$$

To establish the reverse inequality, note homogeneity of the harmonic polynomials

$$
\begin{gathered}
U_{k}(x, \rho)=R_{0}^{k} U_{k}\left(x R_{0}^{-1}, \rho R_{0}^{-1}\right), \\
u_{k}(x, \rho)=R_{0}^{k} u_{k}\left(x R_{0}^{-1}, \rho R_{0}^{-1}\right)
\end{gathered}
$$

in defining the HF by means of a homothetic transformation on (1.2) (1.4) as

$$
\begin{aligned}
& U_{*}(x, \rho)=a_{0}+2 \sum_{k=1}^{\infty} a_{k} R_{0}^{k} U_{k}(x, \rho), \\
& u_{*}(x, \rho)=a_{0}+2 \sum_{k=1}^{\infty} a_{k} R_{0}^{k} u_{k}(x, \rho)
\end{aligned}
$$

in $x^{2}+\rho^{2}<R_{0}^{2}$ so that $I(U)=I_{R_{0}}\left(U_{*}\right)$ and $i(u)=i_{R_{0}}\left(u_{*}\right)$. The inverse operator is normalized by $W^{-1}(1)=1$ and the kernel is nonnegative in $r<R_{0}$. Thus, $u_{*}(x, \rho)=W^{-1}\left[U_{*}(x, \rho)\right] \geqslant I_{R_{0}}\left(U_{*}\right)$ and $i_{R_{0}}\left(u_{*}\right)>I_{R_{0}}\left(U_{*}\right)$. In view of the relation between $U_{*}$ and $U$, and $u_{*}$ and $u$, we find $i(u) \geqslant I(U)$ to conclude

$$
i(u)=I(U) .
$$

Citing [6, p. 147] completes the reasoning. Next consider an extremal property of $I()$ on a subset $\mathcal{H}\left(a_{0}, \ldots, a_{n}\right) \subset \mathcal{H}$ of all those AHF in $\mathcal{H}$ whose first $n+1$ coefficients $a_{0}, \ldots, a_{n}$ are fixed.

THEOREM 2. Let $U \in \mathcal{H}\left(a_{0}, \ldots, a_{n}\right)$ be expanded as in (3.1) and $\lambda_{n}=$ $\lambda_{n}\left(a_{0}, \ldots, a_{n}\right)$ be the smallest eigenvalue of the Toeplitz matrix $T_{n}\left(a_{0}, \ldots, a_{n}\right)$.

Then

$$
I(U) \leqslant \lambda_{n}
$$

and

$$
\operatorname{Sup}\left\{I(u) \mid U \in \mathcal{H}\left(a_{0}, \ldots, a_{n}\right)\right\}=I\left(U_{0}\right)
$$

for unique $U_{0} \in \mathcal{H}\left(a_{0}, \ldots, a_{n}\right)$ expanded as

$$
U_{0}(x, \rho)=2 \lambda_{n}+2 \sum_{k=1}^{m} \mu_{k} V_{k}(x, \rho)-U(0,0),
$$




$$
V_{k}(x, \rho)=\operatorname{Re}\left[\varepsilon_{k} / \sqrt{\left(x-\varepsilon_{k}\right)^{2}+\rho^{2}}\right] .
$$

The principal branch is taken and $\varepsilon_{k},\left|\varepsilon_{k}\right|=1, \mu_{k}>0$ and $1 \leqslant m \leqslant n$ are unique. If $a_{0}^{2}+\cdots+a_{n}^{2}=0$, the extremal function reduces to $U_{0}(x, \rho)=a_{0}$ $=\lambda_{n}$.

Proof. For the set $\mathcal{H}_{*}=\mathscr{H}\left(a_{0}, \ldots, a_{n}\right)$ one associates the set $h_{*}=$ $h\left(a_{0}, \ldots, a_{n}\right)=\left\{W^{-1} U \mid U \in \mathcal{H}_{*}\right\}$. Because of (3.7), the unique extremal function $u_{0} \in \mathcal{K}_{*}$ for $i()$ is mapped by $W$ onto the unique extremal function $U_{0} \in \mathcal{H}_{*}$ for $I()$. The function

$$
U_{0}(x, \rho)=W\left[u_{0}(x, \rho)\right], u_{0}(x, \rho)=\operatorname{Re} f(x+i \rho)
$$

where $f$ is defined by $[6$, p. $151, \# 2]$.

4. Generalization. The corresponding problem of AHF on axisymmetric domains $\Omega$ [8], [9] unlock by mapping the associated axiconvex set $\omega$ into $D$ in a way similar to that in [10]. The Carathéodory-Fejér properties referring to supremum of $\mathrm{HF}$ in $E^{2}$ extend to AHF by replacing inf by sup and interpreting the associates as in [6] while reversing the inequalities. Extension to AHF in $E^{2 \alpha+1}, 2 \alpha+1>0$, follows from operators using the normalized ultraspherical polynomials [1, p. 32].

\section{REFERENCES}

1. R. Askey, Orthogonal polynomials and special functions, Regional Conf. Ser. in Appl. Math., SIAM, Philadelphia, Pennsylvania, 1975.

2. S. Bergman, Integral operators in the theory of linear partial differential equations, Ergebnisse der Math. und Grenzgebiete, Heft 23, Springer-Verlag, New York, 1961.

3. P. Dienes, The Taylor series, Dover, New York, 1957.

4. R. P. Gilbert, Constructive methods for elliptic equations, Lecture Notes in Math., vol. 365, Springer-Verlag, Berlin and New York, 1974.

5. _ Function theoretic methods in partial differential equations, Academic Press, New York, 1969.

6. U. Grenander and G. Szegö, Toeplitz forms and their applications, Univ. of California Press, Berkeley, Calif., 1958.

7. M. Marden, Geometry of polynomials, Math. Surveys, no. 3, Amer. Math. Soc., Providence, Rhode Island, 1966.

8. __ Value distribution of harmonic polynomials in several real variables, Trans. Amer. Math. Soc. 159 (1971), 137-154.

9. P. A. McCoy, Generalized axisymmetric potentials, J. Approximation Theory 15 (1975), 256-266:

10. _ On the zeros of generalized axially symmetric potentials, Proc. Amer. Math. Soc. 61 (1976), 54-58.

11. M. Tsuji, Potential theory in modern function theory, Mauruzen, Tokyo, 1958.

Department of Mathematics, U. S. Naval Academy, Annapolis, Maryland 21401 